\title{
Universidades públicas de la Región Metropolitana: algunas líneas estratégicas de acción para garantizar la inclusión en el contexto de la Pandemia Covid-19
}

\section{Public universities of the Metropolitan Region: some strategic lines of action to guarantee inclusion in the context of the Covid-19 Pandemic}

\section{Matías Causa}

https://orcid.org/0000-0002-6561-8368

causamd@gmail.com

Facultad de Trabajo Social | Universidad

Nacional de La Plata | Argentina

\author{
Karina Fabiana Lastra \\ https://orcid.org/0000-0003-1793-5788 \\ kflastra@gmail.com \\ Escuela de Humanidades | Universidad \\ Nacional de San Martín | Argentina
}

\section{RESUMEN}

En el presente artículo nos proponemos compartir algunas reflexiones preliminares sobre estrategias institucionales inclusivas desplegadas por universidades públicas de la Región Metropolitana con la finalidad de garantizar la continuidad académica de las trayectorias estudiantiles, en el marco de la coyuntura de la no presencialidad en las instituciones y de virtualización de la enseñanza y el aprendizaje debido a la emergencia planteada por la pandemia COVID-19. Para la construcción de los datos, desarrollamos una estrategia metodológica cualitativa en la que se han realizado entrevistas a autoridades, estudiantes, relevamiento de datos estadísticos, consultas de informes, disposiciones y resoluciones administrativas, entre otras fuentes. La indagación de parte de las experiencias institucionales que se encuentran atravesando la Universidad Nacional Arturo Jauretche (UNAJ), Universidad Nacional de Lanús (UNLA), Universidad Nacional de La Plata (UNLP) y Universidad Nacional de San Martín (UNSAM) habilita nuevos y renovados interrogantes que es preciso indagar desde el punto de vista analítico y en el marco de la fuerte interpelación que produjo el referido e inédito contexto. Las líneas estratégicas de acción identificadas refieren a un dinamismo sostenido y actualizado de la agenda de inclusión universitaria al interior de las citadas universidades públicas $y$, a su vez, reconocen los límites de un proceso de democratización que, en el ámbito universitario, por definición, resulta un proceso siempre inacabado y un desafío que recién comienza a develarse (Chiroleu, 2016).

\section{ABSTRACT}

The main purpose of this article is to share some preliminary reflections about the institutional strategies develop by public universities among the Metropolitan Region, as a way to guarantee the academic continuity during the COVID-19 pandemic. In order to build the data, we developed a qualitative methodological strategy composed by interviews with authorities, students, statistical data collection, report inquiries, administrative provisions and resolutions, among other sources. The investigation of the institutional experiences about the National University Arturo Jauretche (UNAJ), National University of Lanús (UNLA), National University of La Plata (UNLP) and the National University of San Martín (UNSAM) leads to new and renewed questions that must be inquired from the analytical point of view and within the contextual framework. The strategic lines of action identified refer to a sustained and updated dynamism of the university inclusion agenda. In addition, the aforementioned universities recognize the limits of a democratization process, always as an unfinished process and a challenge that is just beginning to unfold (Chiroleu, 2016). 


\section{1) UNIVERSIDADES PÚBLICAS DE LA REGIÓN METRO- POLITANA: CONTEXTO POLÍTICO Y SANITARIO.}

La sociedad argentina se encuentra transitando el Aislamiento Social Preventivo y Obligatorio, conocido como ASPO, desde el 20 marzo de 2020. Dicha medida se estableció mediante el Decreto Presidencial 297/2020, siguiendo las recomendaciones de la Organización Mundial de la Salud (OMS), como medida de emergencia por el avance vertiginoso de la Pandemia Covid 19. Anteriormente, mediante la Resolución 108/2020 del Ministerio de Educación, se habían suspendido las clases en los niveles obligatorios del sistema educativo argentino, inicial, primario, secundario y educación superior no universitario. Otra Resolución del Ministerio de Educación, la 104/2020, recomendaba la suspensión de las clases en las universidades. En consonancia con la medida, se suspendieron las clases presenciales y se estableció el dictado de clases remotas, aunque con diferencias devenidas del ejercicio de la autonomía, en algunos casos con continuidad del calendario académico, y en otros con modificaciones.

Si bien el ASPO se extiende sobre todo el territorio nacional, la pandemia impacta sobre áreas geográficas con disparidades sociales y carencias estructurales que le imprimen distinta intensidad a la situación epidemiológica. En efecto, durante el período transitado, las regiones, provincias y ciudades han visto modificar el estado del aislamiento avanzando o retrocediendo en la fase en la cual se habilitan actividades. En el caso de la Región Metropolitana de Buenos Aires, se caracterizó por la persistencia y el crecimiento de los casos positivos de Covid 19, en un territorio extenso y con características de mayor densidad demográfica respecto a otras regiones urbanas del paísi. El informe final del Relevamiento del impacto social de las medidas del aislamiento dispuesto por el PEN (MINCYT, 2020), elaborado a solicitud del Ministerio de Ciencia, Tecnología e Innovación (MINCYT), destacó que las dificultades para cumplir el aislamiento en la Región Metropolitana, se relacionan con 
tres dimensiones, materiales-económicas, actitudinales o de creencia y finalmente vinculada a los hábitos socioculturales. Entre las principales preocupaciones mencionadas como consecuencia del acatamiento al ASPO, figuran las restricciones en la conectividad y el impacto en el seguimiento de las actividades escolares.

En este extenso y muy poblado territorio, se encuentran emplazadas un número importante de universidades públicas, ubicándose algunas en el CPRES Metropolitano y otras en el CPRES Bonaerenseii . Estas universidades públicas fueron creadas en momentos históricos diferentes, pudiendo mencionarse a la Universidad Nacional de La Plata (UNLP) como la de mayor trayectoria e impacto a nivel local y nacional. También se encuentran las llamadas "universidades del conurbano" de la década del noventa hasta las recientes universidades públicas "del Bicentenario", como suele conocerse a las creadas a partir del año 2010iii. En todos los casos, y más allá de las particularidades, comparten demandas y problemas devenidos de los contextos económicos, productivos y sociales de los núcleos habitacionales en los cuales están insertas.

En el presente artículo nos proponemos compartir algunas reflexiones preliminares sobre las líneas de acción institucionales desplegadas por universidades públicas de la Región Metropolitana con la finalidad de garantizar la continuidad académica de las trayectorias estudiantiles, en el marco de la emergencia planteada por la pandemia Covid 19.

\section{En el presente artículo nos proponemos compartir algunas reflexiones preliminares sobre las líneas de acción institucio- nales desplegadas por universidades públicas de la Región Metropolitana con la finalidad de garantizar la continuidad académica de las trayectorias estudiantiles, en el marco de la emergencia planteada por la pandemia Covid 19.}

\section{2) POLÍTICAS INCLUSIVAS EN LA UNIVERSIDAD PÚBLICA ARGENTINA EN EL CONTEXTO DE PANDEMIA COVID 19.}

Con la debida obligación de explicitar a las lectoras y lectores desde qué lugar pretendemos participar del debate, diremos que hacemos nuestros los términos de la posición recientemente asumida por el Consejo Interuniversitario Nacional (CIN, 2020) en la Declaración denominada "Las universidades argentinas frente a la pandemia del COVID-19":

"Las universidades argentinas no renunciamos a nuestro deber de seguir construyendo el futuro de nuestro país con el mejor antídoto para los males del mundo: el derecho social a la educación" 
En otro documento posterior el Consejo Interuniversitario Nacional (CIN, 2020), a los rasgos de excepcionalidad y provisoriedad, agrega que el período actual reviste condiciones cambiantes que imposibilitan prever que las circunstancias vayan a mantenerse iguales durante su permanencia. Así mismo, un informe del Instituto Internacional de la UNESCO para la Educación Superior (IESALC) analiza las medidas destinadas para garantizar la continuidad de la actividad académica y las respuestas institucionales desarrolladas en tal sentido. Entre otras recomendaciones sugiere que el impacto más inmediato es que el cese temporal de las actividades presenciales de las instituciones universitarias ha dejado a las y los estudiantes en una situación totalmente nueva, sin una idea clara de su extensión en el tiempo y cuyas consecuencias son inmediatas sobre su vida cotidiana, los costes y cargas financieras soportados y, por supuesto, la continuidad de sus aprendizaje. No obstante, es preciso destacar que en el presente la gratuidad de la enseñanza (entre otros postulados como la autonomía, el cogobierno, etc.) sigue siendo un elemento crucial que identifica a la universidad pública argentina en el contexto latinoamericano (Atairo y Camou, 2019).

Desde ya, también para el estudiantado las circunstancias implican cambios que requieren capacidades que, en muchos casos, pueden estar poco desarrolladas para adaptarse al tipo de modalidades educativas virtuales. Asimismo, el movimiento estudiantil organizado a través de sus federaciones y agrupacionesiv comenzó a plantear, en el escenario universitario, un conjunto de preocupaciones relativas a las diversas situaciones que atraviesa a la población estudiantil y lograron articular una serie de propuestas para abordar las nuevas dificultades que se presentan de alguna manera alterando la experiencia universitaria estudiantil.

el movimiento estudiantil organizado a través de sus federaciones y agrupaciones comenzó a plantear, en el escenario universitario, un conjunto de preocupaciones relativas a las diversas situaciones que atraviesa a la población estudiantil y lograron articular una serie de propuestas para abordar las nuevas dificultades que se presentan de alguna manera alterando la experiencia universitaria estudiantil.

Por otra parte, desde los sectores del sindicalismo docente universitario también se comenzó a llamar la atención ante el surgimiento de nuevos problemas como la sobrecarga de ocupaciones, los nuevos gastos que recaen en los profesores al poner a disposición las herramientas tecnológicas personales y las conexiones domiciliarias a la red, los incordios y malestares suscitados ante la reorganización de las viviendas para diseñar espacios laborales acordes para la tarea docente, entre otros aspectos. En el mes de junio del presente año la Federación Nacional 
de Docentes Universitarios (CONADU) en reunión paritaria con las autoridades del Ministerio de Educación de la Nación, acordaron un marco regulatorio del trabajo a distancia y las condiciones laborales bajo la situación de excepcionalidad del COVID-19. En el marco del acuerdo las partes reconocen como trabajo docente en contexto virtual o no presencial al que se realiza desde el domicilio de los docentes, o desde otro ámbito sin la presencia de estudiantes, hasta tanto se disponga el restablecimiento de las actividades presenciales en las universidades. Sintéticamente, el acuerdo contempla, entre otros puntos, la regulación de los tiempos de trabajo correspondientes a la carga horaria de los cargos y dedicaciones docentes, el derecho a la desconexión y la pausa virtual, a la percepción del salario correspondiente según los acuerdos paritarios y las condiciones de trabajo establecidas en el Convenio Colectivo de Trabajo, la estabilidad docente y la suspensión de las evaluaciones de reválida de cargos mientras dure la excepcionalidad, la continuidad de la capacitación docente y la formación pedagógica y de enseñanza en entornos virtuales, la disposición de recursos tecnológicos de las universidades, el establecimiento de las plataformas virtuales de las universidades y la provisión de recursos tecnológicos por parte del Ministerio de Educación de la Nación.

A lo largo del texto se busca participar del debate colectivo acerca de las universidades públicas argentinas como instituciones educativas complejas, enfatizando las estrategias institucionales inclusivas desplegadas por universidades públicas de la Región Metropolitana con la finalidad de garantizar la continuidad académica de las trayectorias estudiantiles, la situación de enseñanza virtual a la que se asiste y , más en general, las medidas que se despliegan en un contexto inédito de emergencia y crisis sanitaria a escala global.

\section{3) PROPUESTAS INSTITUCIONALES FRENTE A LA EMERGEN- CIA: UNA MIRADA SOBRE LAS ESTRATEGIAS DESARROLLA- DAS PARA GARANTIZAR LA CONTINUIDAD PEDAGÓGICA EN CUATRO UNIVERSIDADES DE LA REGIÓN.}

La pregunta por las experiencias de las universidades públicas reconoce diversos sentidos y su indagación cobra un renovado interés en función de la modalidad virtual que adquiere la enseñanza como una de las dimensiones de un conjunto más amplio de modificaciones y adecuaciones al que las instituciones universitarias se vieron compelidas en virtud de la actual situación de emergencia sanitaria. El correlato de la pandemia es la no presencia física de los actores universitarios en las sedes de las distintas unidades académicas y la virtualización de la enseñanza.

A partir de una primera aproximación al estudio de cuatro universidades públicas de la Región Metropolitana, nos proponemos recu- 
perar parte de aquellas experiencias institucionales que se encuentran atravesando. Se trata de la Universidad Nacional Arturo Jauretche (UNAJ), Universidad Nacional de Lanús (UNLA), Universidad Nacional de La Plata (UNLP) y Universidad Nacional de San Martín (UNSAM). Como parte del estudio se han realizado entrevistas a autoridades, estudiantes, relevamiento de datos estadísticos, consultas de disposiciones y resoluciones administrativas, entre otras fuentes.

\section{A partir de una primera aproximación al estudio de cuatro universidades públicas de la Región Metropolitana, nos pro- ponemos recuperar parte de aquellas experiencias institucio- nales que se encuentran atravesando. Se trata de la Univer- sidad Nacional Arturo Jauretche (UNAJ), Universidad Nacional de Lanús (UNLA), Universidad Nacional de La Plata (UNLP) y Universidad Nacional de San Martín (UNSAM).}

En estas coordenadas, sostenemos que cobra relevancia la pregunta por las líneas estratégicas de acción que se encuentran desarrollando las universidades públicas en el contexto de la no presencialidad y virtualización de la enseñanza. A continuación se presentan un conjunto de líneas que, sin pretensión de exhaustividad, encontramos centrales en el marco de la fuerte interpelación producida por el inédito contexto de formación no presencial y virtual que atraviesan las universidades. En lo que sigue nos referiremos de manera sucinta a las principales medidas adoptadas por la UNAJ, UNLA, UNLP y UNSAM.

\section{EL CASO DE LA UNAJ}

La UNAJ ha realizado una encuesta docente sobre cursada virtual, con la finalidad de conocer las características, beneficios y dificultades de las cursadas de carácter virtual. Entre los aspectos más destacados figuran la disponibilidad en el uso del dispositivo de trabajo (notebook, PC, celular) el $66 \%$ de los encuestados respondió que es de uso exclusivo, mientras que el $34 \%$ restante debe compartirlo con alguien del grupo familiar. Sobre el espacio de trabajo adecuado para la actividad laboral el $62 \%$ considera que dispone de un espacio adecuado, mientra que el $38 \%$ no cuenta con él. Respecto a las principales dificultades sobre la virtualidad manifestadas por las y los docentes, las respuestas se concentran en torno a la falta de tiempo para la preparación de las clases en términos de actividades y el seguimiento y la corrección, representa el $52,9 \%$ del total de las preocupaciones. Sólo un $16,4 \%$ considera que le falta capacitación docente para el uso tecnológico del campus, mientras que un $5 \%$ considera como una gran dificultad la falta de trabajo en equipo y un $8,2 \%$ cree que no hay dificultades. Finalmente, se consultó 
respecto a la posibilidad de continuidad de la enseñanza virtual luego del ASPO, un 66,7 \% de las y los docentes considera que sí debe persistir, un $16,1 \%$ considera que no, y un $17,1 \%$ no tiene opinión formada sobre el tema. En el caso de la UNAJ el área de virtualización no estaba desarrollada, con lo cual en marzo hubo que organizar toda la estructura de la plataforma Moodle, en este caso de carácter centralizado, para poder avanzar con las clases virtuales. Los docentes mayoritariamente no contaban con capacitación (Biblioteca central), con lo cual se pusieron a disposición orientaciones para el uso de las diversas plataformas para videoconferencias a través de tutoriales. Aunque no se menciona en la encuesta, las autoridades entrevistadas destacaron la muy buena disposición de los equipos docentes para adecuarse a la emergencia planteada por la virtualización, a la vez que señalan el impacto emocional manifestado como consecuencia del aislamiento.

\section{Con relación a las y los estudiantes, para garantizar el acompa- ñamiento la UNAJ estableció becas de ayuda económica para conectividad destinadas a estudiantes regulares (al menos 1 materia), con ingresos del grupo familiar inferiores a tres sala- rios mínimos y que no cuenten con PROGRESAR.}

Con relación a las y los estudiantes, para garantizar el acompañamiento la UNAJ estableció becas de ayuda económica para conectividad destinadas a estudiantes regulares (al menos 1 materia), con ingresos del grupo familiar inferiores a tres salarios mínimos y que no cuenten con PROGRESAR. Se extiende durante el primer cuatrimestre y se renueva automáticamente si continúa el ASPO, con una asignación mensual de $\$ 500$. Por otra parte, se asignaron tarjetas SIMs M2M con 3 gigabytes de datos para navegar y 1000 SMS mensuales. Los requisitos para acceder son similares a la Beca de conectividad, al igual que su renovación. A nivel comunitario, se puso en marcha el Voluntariado Covid 19, que contó con 3000 inscriptos en su etapa inicial y cerca de 1000 en la segunda convocatoria. Las y los estudiantes participaron junto a docentes y técnicos del Hospital El Cruce de la instalación y puesta en marcha de hospitales modulares. También participan en la atención del call center de emergencias en los municipios y asisten a los centros de salud para realizar triage.

\section{EL CASO DE LA UNLA}

En la UNLA, las autoridades entrevistadas señalan que previo a la pandemia el Campus Virtual funcionaba como apoyo a las clases presenciales, pero no era una herramienta usada por todas las materias. Ante la irrupción del ASPO, se trabajó en generar un aula virtual para 
cada asignatura para sostener la continuidad académica de cerca de 14.000 estudiantes durante el primer cuatrimestre. También se aumentó la capacidad del servidor para poder optimizar el funcionamiento digital. Las aulas virtuales cuentan con una amplia variedad de herramientas y recursos tecnológicos. Para los docentes se desarrollaron talleres específicos de capacitación sobre la Plataforma Moodle, tutoriales y atención personalizada para quienes lo necesitaban. Coordinadas por el equipo del campus virtual se crearon aulas de asistencia a la virtualidad y se realizaron capacitaciones por videoconferencia. Aun así, las fuentes consultadas señalan que para los equipos docentes la virtualización de las asignaturas en estas condiciones demandó mucho tiempo de trabajo, a la vez que no todos los docentes cuentan con equipamiento tecnológico propio o bien que este debe ser compartido con otras personas de la familia.

\section{Ante la irrupción del ASPO, se trabajó en generar un aula vir- tual para cada asignatura para sostener la continuidad acadé- mica de cerca de 14.000 estudiantes durante el primer cuatri- mestre. También se aumentó la capacidad del servidor para poder optimizar el funcionamiento digital.}

En términos territoriales, la UNLA desarrolló una serie de programas de voluntariado por iniciativa propia: de asistencia alimentaria a comedores cercanos a la Universidad y de acompañamiento a familias en situaciones de vulnerabilidad fuertemente afectadas por la pandemia. Asimismo, en convenio con el Ministerio de Salud de la Provincia de Buenos Aires se están generando tres acciones específicas: Seguimiento a través de técnicas virtuales a pacientes con COVID-19 y contactos estrechos y detección de sus necesidades; atención remota de la situación sanitaria de las residencias geriátricas de la zona; contención psicológica a las personas que están trabajando en el marco de los voluntariados. Por otra parte, se relevó el estudiantado con dificultades en la conectividad y se amplió el criterio de becas incorporando becas de conectividad.

\section{EL CASO DE LA UNLP}

En el caso de la UNLP, desde el 15/3/2020 y extendiéndose luego en consonancia con las políticas adoptadas por el Gobierno Nacional, se estableció la no realización de instancias áulicas presenciales de enseñanza en todo el ámbito de la institución, asignando atribuciones excepcionales a las autoridades para el mejor desenvolvimiento de las funciones universitarias esenciales en el marco de la crisis sanitaria. Además, se estipularon sistemas de equivalencias entre horas acreditadas de voluntariado realizadas con motivo de la emergencia sanitaria y las que corresponden a instancias prácticas de las carreras de grado, en la 
medida en que exista una razonable correlación entre unas y otras.

La UNLP ratificó la vigencia de los calendarios académicos establecidos, con las adecuaciones específicas que cada facultad y/o colegio secundario estime conveniente realizar en el marco de la emergencia sanitaria provocada por la pandemia de COVID-19. En tal sentido, se mantuvieron las cursadas en modalidad a distancia, y los mecanismos de evaluación según las modalidades y posibilidades de cada dependencia. Así, la UNLP diseñó y puso en marcha en tiempo récord un programa especial de educación a distancia para que las y los docentes puedan virtualizar sus clases y garantizar, de este modo, el acceso de los estudiantes a los contenidos educativos.

\section{La UNLP ratificó la vigencia de los calendarios académicos es- tablecidos, con las adecuaciones específicas que cada facultad y/o colegio secundario estime conveniente realizar en el mar- co de la emergencia sanitaria provocada por la pandemia de COVID-19. En tal sentido, se mantuvieron las cursadas en mo- dalidad a distancia, y los mecanismos de evaluación según las modalidades y posibilidades de cada dependencia.}

A poco de haber transcurrido 30 días, más de 2.000 cátedras de las facultades y colegios comenzaron a dictar clases virtuales. El "Programa de apoyo a la Educación a Distancia para Facultades y Colegios en el contexto de la pandemia de COVID-19" (PAED) comenzó a funcionar el 16 de marzo, luego que la UNLP resolviera suspender todas las actividades académicas presenciales. La iniciativa, elaborada en el ámbito de la Secretaría Académica a través de la Dirección General de Educación a Distancia y Tecnologías, buscó garantizar la cantidad y calidad de los contenidos a disposición de alrededor de 95 mil estudiantes, y sostener la continuidad del desarrollo de las cursadas a lo largo del año. En lo que refiere a las evaluaciones, la UNLP enfatizó que debe tenerse en cuenta la adecuada armonización del derecho de las y los estudiantes a ser evaluados con el necesario encuadre que permita a los docentes el ejercicio de las evaluaciones.

Con relación a medidas de bienestar estudiantil, la UNLP creó en el ámbito de la Prosecretaría de Bienestar Universitario y en el marco del Programa Igualdad de Oportunidades para Estudiar, el Subprograma denominado "Beca TU PC para estudiar". Mediante esta beca se entregarán a los estudiantes que lo requieran, en comodato, una PC de escritorio (monitor, CPU, teclado, mouse), una Notebook o Tablet, según corresponda, durante el período en el que transcurran sus estudios de grado.

Por último, con relación a las y los docentes, incluyendo los colegios secundarios, la universidad cuenta con aproximadamente 4.500 personas trabajando a distancia con sus estudiantes y 1.500 estuvieron -y continúan- participando de propuestas de capacitación en la op- 
ción pedagógica a distancia. Por otra parte, en un informe reciente la Asociación de Docentes Universitarios de la Universidad Nacional de La Plata (ADULP) llevó a cabo, a través de su Centro de Estudios en Trabajo y Universidad Secretaría de Formación, un relevamiento sobre las condiciones y medio ambiente de trabajo en contexto de ASPO en la UNLP. El informe concluye que sobre una estructura desigual preexistente de los puestos de trabajo por cargo o dedicación se instalan las vicisitudes del trabajo docente virtualv.

\section{EL CASO DE LA UNSAM}

En la UNSAM, el relevamiento a docentes realizado por la Secretaría Académica aún se encuentra en proceso de sistematización. El proceso de virtualización de la enseñanza se concretó a partir de la creación de aulas virtuales, pero considerando la heterogeneidad propia de las unidades académicas, respetando en cada caso las culturas digitales preexistentes a la pandemia. Para acompañar a los docentes en este experiencia de aprendizaje y evaluación remota, se creó desde la Secretaría Académica un micrositio en el cual se despliegan modelos, ejemplos, e instructivos. En este espacio se le concede amplia importancia a la evaluación de los aprendizajes en la cursada virtual, pero también al diseño de propuestas para los exámenes finales, ya que por Resolución Rectoral (RR 458-2020), se encomendó a las unidades académicas implementar mecanismos de acreditación de conocimientos finales de cada unidad curricular a distancia, es decir exámenes finales y regímenes de promoción directa. Como parte de la dinámica de este proceso, las autoridades del área consideran que se abren algunas preguntas de investigación vinculadas a la reflexividad de las y los docentes en el pasaje de la modalidad presencial a virtual, en torno al diseño de los materiales y las propuestas didácticas.

Por su parte, de manera anual la UNSAM realiza una convocatoria a través del Sistema de Becas de Apoyo Económico, mediante el cual se otorgan becas de ingreso, becas de continuidad y becas de excelencia a las y los estudiantes. Este año, se decidió no otorgar las Becas de Excelencia y ampliar el número de beneficiarios de las Becas de Ingreso y de las Becas de Continuidad, priorizando de este modo el acceso y la permanencia. A su vez, la Secretaría Académica y la Secretaría de Desarrollo Social del Municipio de San Martín acordaron un plan de asistencia alimentaria y escolar a las familias de las y los estudiantes de la Escuela Secundaria Técnica de la UNSAM. Se diseñó una dieta saludable que incluye material didáctico y permite sostener el vínculo pedagógico. Finalmente el dictado de clases en el Centro Universitario San Martín (CUSAM), que funciona en la Unidad Penal $N^{\circ} 48$ del Servicio Penitenciario Bonaerense, continuó a partir del uso de dispositivos móviles y con la entrega de material bibliográfico fotocopiado. 
Es evidente la importancia de las políticas inclusivas en la concreción del Derecho a la Educación Superior, ya que, la masificación que se produjo a escala global en el nivel a partir de las últimas décadas del siglo XX, no garantiza por sí misma una mejora en la participación de los sectores sociales de menores ingresos. Sin embargo, varios estudios e investigaciones señalan como relevante la expansión producida en América Latina y consideran que se ha experimentado un importante avance en la región, ya que se redujo la desigualdad social en el ingreso (Ferreyra, Avitabile, Botero Álvarez, Haimovich Paz y Urzúa, 2017). En un trabajo reciente A. Ezcurra (2019), considera que hubo un decrecimiento de la desigualdad en la admisión, y propone como hipótesis a explorar si esa aceleración del ingreso al nivel, se da como consecuencia de políticas gubernamentales orientadas a la democratización. Todo esto en el marco del fomento de políticas públicas que en algunos casos ampliaron la oferta estatal a través de la creación de nuevas instituciones, pero también dieron un fuerte impulso a los programas de becas y apoyo económico para sectores socialmente desfavorecidos (Chiroleu y Marquina, 2017; García de Fanelli y Adrogué, 2019).

De modo complementario, otros estudios han observado la responsabilidad institucional en el desarrollo de políticas y programas específicos para el acompañamiento de las trayectorias estudiantiles. En este sentido A. Cambours de Donini (2018) propone pensar la inclusión en la universidad en distintos sentidos que incluyan la necesidad de políticas específicas que contemplen la situación de los grupos de estudiantes provenientes de sectores tradicionalmente excluidos y a su vez propone no limitar esas políticas a la dimensión socioeconómica, sino que sugiere incorporar el carácter dinámico de las culturas juveniles, aunque, como señala, no son sólo jóvenes quienes ingresan a estas universidades. Finalmente acordamos con la siguiente definición de políticas institucionales inclusivas:

(....)Optamos por denominar políticas institucionales inclusivas a aquellos mecanismos y estrategias encaminadas a generar condiciones para igualar resultados en las trayectorias académicas de la población estudiantil proveniente de sectores sociales desfavorecidos. El concepto de inclusión supone un reconocimiento de la diversidad y la necesidad de pertinencia de la oferta curricular y el acompañamiento institucional, a diferencia de "la igualdad de oportunidades" que supone una sociedad con puntos de partida homogéneos y las "políticas compensatorias" que suponen un déficit que puede ocasionar bajas expectativas y circuitos educativos devaluados (Cambours de Donini, Lastra, Mihal y Muiños de Britos, 2019:4). 
De acuerdo a las indagaciones realizadas hasta el momento y de modo preliminar, podemos identificar la presencia de líneas de acción inclusivas en las universidades públicas estudiadas. Es interesante observar cómo aun cuando se trata de instituciones de distinta trayectoria respecto a su creación e historia, demuestran perseverancia en un ideario común: el reconocimiento del derecho a la educación superior y la vinculación con las demandas sociales y territoriales, de modo general y en particular, planteadas como consecuencia de la emergencia por la pandemia.

Como parte de las preocupaciones por adecuar la continuidad del calendario académico con los actores institucionales, se han desarrollado relevamientos tendientes a identificar condiciones de trabajo docente remoto durante el ASPO, pudiendo identificarse algunos temas de modo persistente. En primer lugar, planteamos que es posible identificar en los relatos de las y los docentes su preocupación ante la virtualización abrupta e intempestiva que debieron experimentar sus propuestas pedagógicas, la falta de capacitación para enseñar a través de plataformas virtuales o la formación acelerada que debieron asumir a la par que dictan sus cursos, la poca claridad en cuanto a las evaluaciones y el grado de participación y aprendizaje del estudiantado. Preocupaciones que en rigor también se combinan con el rediseño permanente que realizan de las estrategias pedagógicas y con el compromiso político que implica transmitir mensajes inclusivos y esperanzadores a los estudiantes, fundamentalmente a aquellos que transitan los primeros años de la formación universitaria. Como apunta Larraguivel:

...los maestros universitarios no sólo se han visto en la necesidad de aprender en el aislamiento los mecanismos técnicos de la educación virtual, sino que muy probablemente también se encuentren experimentando procesos de adaptación a las nuevas situaciones que impone la educación en línea, en especial si partimos del hecho de que la efectividad de la enseñanza en el salón de clases se apoya en una buena interacción social entre profesor y alumno (2020:110).

planteamos que es posible identificar en los relatos de las y los docentes su preocupación ante la virtualización abrupta e intempestiva que debieron experimentar sus propuestas pedagógicas, la falta de capacitación para enseñar a través de plataformas virtuales o la formación acelerada que debieron asumir a la par que dictan sus cursos, la poca claridad en cuanto a las evaluaciones y el grado de participación y aprendizaje del estudiantado. 
En el marco del ASPO, las áreas de bienestar estudiantil de las universidades públicas intensificaron sus acciones con la finalidad de atender las demandas y las dificultades manifestadas por las y los estudiantes de la región. En este sentido, se puede observar que las líneas de acción incluyen desde ampliación de las becas con fines específicos hasta trabajos comunitarios en articulación con organizaciones territoriales. Es preciso considerar que, en este contexto, existe un mayor riesgo de interrupción y abandono de la universidad que podría incrementarse debido a la situación socioeconómica del país, tanto durante como una vez concluida la pandemia. Estos posibles efectos variarán entre los grupos de estudiantes de acuerdo con sus características socioeconómicas y condiciones materiales, de sus habilidades y capacidades de aprendizaje, de las áreas de conocimiento de la carrera de pertenencia (la situación de las prácticas de formación en terreno en el caso de las carreras que las incluyen en sus planes de estudio amerita un análisis específico), y de los apoyos que les brinden tanto las instituciones universitarias como las y los docentes.

Respecto a esto, en el citado informe de la IESALC-UNESCO, se considera que es muy probable un retraimiento en la demanda en la oferta de educación superior, sin embargo para el caso de las universidades públicas estudiadas y aún en contextos de provisoriedad, algunos indicios señalan que ha habido mayores y mejores niveles de retención del estudiantado como así también, en algunos casos, la inscripción a las cursadas ha superado los porcentajes habituales. En este sentido, una autoridad entrevistada nos señalaba que tuvieron que "poner un cupo en la inscripción a algunas materias ya que desbordaba la capacidad del equipo docente". En otra institución, mencionaban el malestar docente asociado a "la cantidad de inscriptos" y la imposibilidad de realizar nuevas designaciones por las limitaciones presupuestarias.

\section{en el citado informe de la IESALC-UNESCO, se considera que es muy probable un retraimiento en la demanda en la oferta de educación superior, sin embargo para el caso de las universi- dades públicas estudiadas y aún en contextos de provisorie- dad, algunos indicios señalan que ha habido mayores y mejo- res niveles de retención del estudiantado como así también, en algunos casos, la inscripción a las cursadas ha superado los porcentajes habituales.}

Por último, pensamos que el recorrido efectuado habilita la pregunta respecto a cuáles podrán ser los efectos educativos de mediano y largo plazo que esta realidad traiga consigo en una posible reestructuración del sistema universitario y particularmente en el tipo de ofertas de carreras universitarias de grado. En este punto conviene recordar que, en términos de condiciones de partida y de acuerdo a la informa- 
ción recientemente publicada por la SPU (2020), actualmente existen en el país 392 ofertas de carreras con modalidad de estudio a distancia (192 en el sector privado y 200 en el sector estatal) y 161.281 estudiantes que cursan bajo esta modalidad (más del $60 \%$ de ellos pertenecen al sector de gestión privado). Del mismo modo, algunos autores sugieren pensar las potencialidades de la educación virtual ya sea como "complementaria" de la instancia presencial, o bien como oportunidad para alcanzar horizontes geográficos excluidos. (Veiravé, 2020). 


\section{REFERENCIAS BIBLIOGRÁFICAS}

ADULP (2020). “Primer Informe. Relevamiento sobre condiciones y medio ambiente de trabajo en contexto de ASPO en la UNLP". Centro de Estudios en Trabajo y Universidad Secretaría de Formación.

Atairo, Daniela.; Camou, Antonio. (2019). "Las dos caras de Jano. Cogobierno y gratuidad en las universidades latinoamericanas". En: La conquista de un derecho: reflexiones latinoamericanas a 70 años de la gratuidad universitaria en Argentina / Graciela Giménez ... [et al.]; compilado por Ernesto Villanueva;-1a ed . - Ciudad Autónoma de Buenos Aires: CLACSO; Quilmes: Universidad Nacional de Quilmes; Florencio Varela: Universidad Nacional Arturo Jauretche.

Cambours de Donini, Ana; Lastra, Karina; Mihal, Ivana; Maris Muiños de Britos, Stella (2019). "Límites y posibilidades de las políticas institucionales bonaerense. Explorando caminos nuevos". Revista de Sociología de la Educación-RASE, 12 (2), 301-317. http://dx.doi.org/10.7203/RASE.12.2.14667

Cambours de Donini, Ana María (2018). "Políticas institucionales para favorecer la retención en los primeros años de la vida universitaria". En: Pogré, $P$ et al (coord.) (2018). Los inicios de la vida universitaria: políticas, prácticas y estrategias para garantizar el derecho a la educación superior. Teseo, Buenos Aires.

Chiroleu, Adriana. (2016). "Políticas públicas de inclusión y democratización universitaria: hacia una ampliación de las bases sociales de las instituciones". En: Aranciaga, I. "La universidad y el desafío de construir sociedades inclusivas: debates y propuestas sobre modelos universitarios desde una perspectiva comparativa" / Ignacio Aranciaga ; compilado por Ignacio Aranciaga. - 1a ed . - Río Gallegos: Universidad Nacional de la Patagonia Austral.

Chiroleu, Adriana y Marquina, Mónica (2017). “Democratisation or credentialism? Public policies of expansión of higher education en Latin America". Policy Reviews in Higher Education., 1 (2), pp.139-160

Consejo Interuniversitario Nacional. (2020). Las universidades argentinas frente a la pandemia del COVID 19.

Consejo Interuniversitario Nacional. (2020). "Algunas cuestiones normativas relacionadas con decisiones sobre validez, evaluación, acreditación y dictado de las carreras en las instituciones universitarias públicas durante el actual período de emergencia sanitaria". Documento Anexo del Acuerdo Plenario No 1103/20.

Decreto Aislamiento Social Preventivo y Obligatorio. (2020). Decreto Presidencial N²297/2020. Boletín Oficial de la República Argentina.

Ezcurra, Ana María. (2019). "Educación superior: una masificación que incluye y desiguala". En: Ezcurra A. M. (comp.). Derecho a la Educación: Expan- 
sión y desigualdad: tendencias y políticas en Argentina y América Latina. Saenz Peña. Universidad Nacional de Tres de Febrero. Libro digital.

Fernández, L. (2011). Censo 2010. Somos 14.819.137 habitantes en la Región Metropolitana de Buenos Aires. Instituto del Conurbano. Universidad Nacional de General Sarmiento.

Ferreyra, María Marta, Avitabile, Ciro, Botero Álvarez, Javier, Haimovich Paz, Francisco y Urzúa, Sergio (2017). "At a Crossroads: Higher Education in Latin America and the Caribbean". Washington, DC: Directions in DevelopmentHuman Development; World Bank.

García de Fanelli, Ana. y Adrogué, Cecilia. (2019). "Equidad en el acceso y la graduación en la Educación Superior: Reflexiones desde el Cono Sur". Archivos Analíticos de Políticas Educativas, 27(96). https://doi.org/10.14507/ epaa. 27.3843

IESALC-UNESCO. (2020). Covid 19 y Educación Superior. De los efectos inmediatos al día después. Análisis de impactos, respuestas políticas y recomendaciones. Caracas. UNESCO-IESALC.

Larraguivel, Estela. (2020). "La práctica docente universitaria en ambientes de educación a distancia. Tensiones y experiencias de cambio". En: IISUE. Educación y pandemia. Una visión académica, México, UNAM. Disponible en: http://www.iisue.unam.mx/nosotros/covid/educacion-y-pandemia (Consultado el 25/5/2020).

Ministerio de Ciencia, Tecnología e Innovación. (2020). Relevamiento del impacto social de las medidas del aislamiento dispuestas por el PEN. Comisión de Ciencias Sociales de la Unidad Coronavirus 2019.

Ministerio de Educación, República Argentina. (2020). Resolución º 108/2020. Boletín Oficial de la República Argentina.

Ministerio de Educación, República Argentina (2020). Resolución Nº 104/2020. Boletín Oficial de la República Argentina.

Secretaría de Políticas Universitarias (SPU). (2020). Síntesis de información de estadísticas universitarias 2018-2019. Dirección Nacional de Presupuesto e Información Universitaria. Disponible en: https://www.argentina.gob.ar/ sites/default/files/sintesis_20182019_sistema_universitario_argentino_-_ ver_final_1_0.pdf

Veiravé, Delfina (2020). “Esta pandemia puso en la opinión pública el rol fundamental de la ciencia y el aporte de nuestras investigadoras e investigadores...." Integración y Conocimiento, 9(2), 101 - 107. Recuperado a partir de https://revistas.unc.edu.ar/index.php/integracionyconocimiento/article/ view/29546 
Universidad Nacional Arturo Jauretche. Centro de Política Educativa. (2020). Encuesta a docentes sobre cursada virtual. Primer cuatrimestre 2020.

Universidad Nacional de San Martín (2020). Resolución Rectoral 458/2020. Modalidad de Educación a Distancia.

\section{CITAS}

¡ La Región Metropolitana de Buenos Aires se extiende en 13.947 km2 con una población total de 14.839.746 y una densidad poblacional de 6.105 (hab/ km2). Está compuesta por la Ciudad de Buenos Aires, y 40 partidos entre los 24 del Gran Buenos Aires, y 16 restantes de la denominada gran corona, que incluye el Gran La Plata.

ii El sistema de Educación Superior de Argentina está organizado regionalmente para facilitar la planificación y articulación. Las regiones son siete y cada una está coordinada por un Consejo Regional de la Educación Superior (CEPRES), integrado por los ministros de educación de las provincias comprendidas en la región y los rectores de las universidades, públicas y privadas.

iii La lista de universidades públicas contempla también a las Universidades Nacionales de Luján y Lomas de Zamora, creadas en el año 1972. Mientras que las llamadas "universidades del conurbano" creadas en la década del noventa se refiere a las Universidades Nacionales de Lanús, La Matanza, Quilmes, General Sarmiento, San Martín y Tres de Febrero. Finalmente está el grupo de las cinco "universidades del Bicentenario" con fecha de creación en el año 2009 y con sede en el conurbano y con la siguiente denominación: Universidad Nacional de Avellaneda, Del Oeste (con sede en Merlo), Arturo Jauretche (con sede en Florencio Varela), José C. Paz y Moreno.

iv En el caso de la UNLP, para mayor información véase: https://www.diariocontexto.com.ar/2020/05/21/la-fulp-destaco-la-entrega-de-computadoras-de-la-unlp-a-estudiantes/ (Consultado el 10/6/2020).

v Con el objetivo de describir el estado del trabajo docente en el contexto actual, identificar situaciones de vulneración de derechos, y visibilizar desigualdades, el informe detalla que sobre la apropiación de la tarea, el 97,5\% de las y los docentes encuestados (1.620) virtualizó las tareas pedagógicas. Además, se agrega que la mayoría de los docentes dicen que trabajan más que antes, que tienen más tareas que antes, que no sabían casi nada o poco sobre enseñanza virtual, y que no disponen de espacio, escritorio y silla, que comparten computadora equipada, o que no estaba equipada y tuvieron que gastar dinero en hacerlo comprando cámaras, micrófonos, pizarras o la computadora completa. 